\title{
Clinical Efficacy Analysis on Total Hip Arthroplasty with Different Surgical Approaches
}

\author{
Weiwei Wang ${ }^{1}$, Hongkai Lian ${ }^{2, *}$ \\ ${ }^{1}$ The Second Affiliated Hospital of Zhengzhou University, Henan, China \\ ${ }^{2}$ Zhengzhou Central Hospital, Henan, China \\ *Corresponding author: lianhongkaidavid@163.com
}

Keywords: Anterolateral approach; Posterolateral approach; Total hip arthroplasty; Efficacy.

\begin{abstract}
Objective: To observe and analyze the clinical efficacy of total hip arthroplasty with different surgical approaches. Methods: From August 2019 to August 2020, 82 patients with total hip arthroplasty in the hospital were selected as the observation objects. Based on the numerical random table method, they were divided into two control groups: conventional group (total hip arthroplasty via posterolateral approach) and study group (total hip arthroplasty via anterolateral approach), with 41 patients in each group. Results: Although the operation time of the study group was longer than that of the conventional group, the operation incision was smaller than that of the conventional group. Also, the operation bleeding volume was lower than that of the conventional group, and the hospital time was shorter than that of the conventional group $(\mathrm{P}<0.05)$. Harris hip function scores at the third, sixth and twelfth months of surgical treatment in the study group were higher than those in the conventional group $(\mathrm{P}<0.05)$. Surgical complications in the study group were less than those in the conventional group $(\mathrm{P}<0.05)$. Conclusion: Compared with the posterolateral approach, anterolateral approach to total hip arthroplasty is more minimally invasive, with many advantages such as less bleeding, faster postoperative recovery, higher safety, fewer complications, etc. It can also significantly improve the patients' hip function, which is worthy of attention in clinical practice and widespread promotion.
\end{abstract}

\section{Introduction}

Total hip arthroplasty is relatively common in clinical practice, and it is usually used to treat patients with femoral neck fracture and hip joint diseases. It can not only help patients get rid of the disease issues, but also help patients recover their walking ability [1]. Currently, there are many approaches for this type of surgery. In the past, the posterolateral approach was generally adopted, which was observed more clearly, exposed more fully and was convenient for prosthesis implantation. However, the posterolateral approach required a large incision, which caused great damage to patients and required a long recovery time after operation. Under the posterolateral approach, some muscles and posterior joints behind the hip joint need to be removed, which may lead to prosthesis dislocation [2]. In recent two years, with the deepening of clinical research and the widespread popularization of minimally invasive concept, anterolateral approach to total hip arthroplasty is increasingly popular in clinical practice. It is not only minimally invasive and less blood loss, but also can further accelerate the patients' recovery process [3]. Accordingly, this study compared and analyzed the application value of anterolateral approach and posterolateral approach in 82 patients undergoing total hip arthroplasty for clinical reference.

\section{General Information and Methods}

\subsection{General information}

In this study, 82 patients with total hip arthroplasty were received from August 2019 to August 2020. Inclusion criteria: Garden classification of femoral neck fracture type III-IV, first time 
replacement, BMI $<30 \mathrm{~kg} / \mathrm{m}^{2}$ and research letter signed with the consent of the parties. Exclusion criteria: cardiovascular disease, brain organic lesions, revision of secondary total hip arthroplasty, intolerance of surgical anesthesia and surgical contraindications. According to the requirements of randomized controlled trials and numerical random table method, the observation subjects were divided into two control groups: conventional group and study group, with 41 cases in each group. The study group consisted of 22 males and 19 females, and aged 61-77 years, with an average age of 69.6 \pm 3.9 years. Among them, 13 cases were femoral neck fracture, and 13 cases were femoral head necrosis and 15 cases were osteoarthritis. The conventional group consisted of 20 males and 21 females, and aged 61-76 years, with an average age of 69.5 \pm 4.1 years. Among them, 14 cases were femoral neck fracture, and 13 cases were femoral head necrosis and 14 cases were osteoarthritis. The basic data in each group were tested by clinical statistics, and the result was $p>0.05$. This study is in accordance with the requirements of "Helsinki Declaration of the World Medical Association".

\subsection{Methods}

Anterolateral approach was given to 41 patients in the study group: combined lumbar and epidural anesthesia was performed, and the healthy lateral position was maintained. The incision from the anterior margin of the great trochanter extended to about $8 \mathrm{~cm}$ behind the anterior superior iliac spine, followed the gap between the gluteus medius and the tensor fascia latae to fully expose the anterior joint capsule. The joint capsule was cut open, and then the prosthesis was placed.

Posterolateral approach was given to 41 patients in the conventional group: combined lumbar and epidural anesthesia was performed, and the healthy lateral position was maintained. The incision was located at $1 / 3$ of the line between the greater trochanter and the posterior superior iliac crest, and was continuously extended along the lateral femoral axis to fully expose the greater trochanter and separate carefully, thus avoiding sciatic nerve injury. The proximal 1 / 3 quadratus femoris muscle was cut off, and the circumflex tendon was cut off at a distance of $1 \mathrm{~cm}$ from the greater trochanter, so that the joint capsule was exposed. The joint capsule was cut, and then the prosthesis was placed.

Routine anticoagulant treatment was performed postoperatively, and the wound drainage tube was removed after 24 hours. If the patient's condition allowed, the patients were guided by rehabilitation doctors to raise their straight legs and bend their hips. X-ray was used to observe the recovery of hip joint at $72 \mathrm{~h}$ after surgery, and weight-bearing exercise was tried under the guidance of medical staff. Patients were encouraged to walk on crutches 3 weeks after surgery, and encouraged to exercise with full weight at 5 weeks after surgery.

\subsection{Observation indicators}

The operation time, incision size, blood loss and hospital stay were observed and recorded. The patients were observed for complications such as incision infection, venous embolism of lower limbs, prosthesis dislocation, etc. Regular follow-up and X-ray reexamination were conducted before operation and at the third, sixth and twelfth months after surgery. Harris hip joint function score was used to evaluate the recovery of patients' hip joint function. The higher the score, the better the recovery effect.

\subsection{Data statistics processing}

The counting data and measurement data obtained in this study were imported into SPSS22.0 statistical software for data processing. t-test was used to test the $(\mathrm{x} \pm \mathrm{s})$ measurement data with normal distribution, and the [n (\%)] count data expressed by chi-square test percentage or constituent ratio. The difference between groups was determined by $\mathrm{P}$ value, and the test level $\alpha$ was set to 0.05 . Then, the statistical significance judgment standard was mainly $(\mathrm{P}<0.05)$. 


\section{Results}

3.1 The operative performance of study group and conventional group is shown in Table 1

Table 1. Comparison of surgical indicators between the study group and the conventional group

\begin{tabular}{llllll}
\hline Groups & Cases & $\begin{array}{l}\text { Operation } \\
\text { time }(\mathbf{m i n})\end{array}$ & $\begin{array}{l}\text { Surgical } \\
\text { incision }(\mathbf{c m})\end{array}$ & Blood loss (ml) & $\begin{array}{l}\text { Hospital stay } \\
(\mathbf{d})\end{array}$ \\
\hline $\begin{array}{l}\text { Study group } \\
\text { Conventional }\end{array}$ & 41 & $77.8 \pm 15.9$ & $7.1 \pm 0.7$ & $288.6 \pm 55.4$ & $11.8 \pm 2.2$ \\
group & 41 & $70.4 \pm 15.6$ & $15.6 \pm 2.5$ & $420.4 \pm 70.5$ & $16.9 \pm 1.7$ \\
t & - & 6.038 & 18.346 & 18.920 & 7.236 \\
p & - & $<0.05$ & $<0.05$ & $<0.05$ & $<0.05$ \\
\hline
\end{tabular}

3.2 The Harris scores between the study group and the conventional group before and after surgery are shown in Table 2

Table 2. Comparison of Harris scores between study group and conventional group before and after surgery $(\mathrm{x} \pm \mathrm{s})$

\begin{tabular}{lllllll}
\hline Groups & Cases & $\begin{array}{l}\text { Before } \\
\text { surgery }\end{array}$ & $\begin{array}{l}\text { 3 months } \\
\text { after surgery }\end{array}$ & $\begin{array}{l}\text { 6 months } \\
\text { after surgery }\end{array}$ & $\begin{array}{l}\text { 12 months } \\
\text { after surgery }\end{array}$ \\
\hline $\begin{array}{l}\text { Study group } \\
\text { Conventional }\end{array}$ & 41 & $35.6 \pm 6.6$ & $89.8 \pm 6.2$ & $92.5 \pm 5.7$ & $96.4 \pm 7.3$ \\
group & 41 & $35.7 \pm 6.9$ & $80.5 \pm 5.5$ & $85.4 \pm 7.6$ & $87.1 \pm 6.3$ \\
$\mathrm{X}^{2}$ & - & 0.384 & 6.145 & 8.565 & 7.354 \\
$\mathrm{p}$ & - & $<0.05$ & $<0.05$ & $<0.05$ & $<0.05$ \\
\hline
\end{tabular}

3.3 Complications associated with surgery in the study group and the conventional group are shown in Table 3

Table 3. Comparison of surgical complications in study group and conventional group

\begin{tabular}{llllll}
\hline Groups & Cases & $\begin{array}{l}\text { Incision } \\
\text { infection }\end{array}$ & $\begin{array}{l}\text { Lower extremity } \\
\text { venous embolism }\end{array}$ & $\begin{array}{l}\text { Prosthetic } \\
\text { dislocation }\end{array}$ & $\begin{array}{l}\text { Total } \\
\text { incidence }\end{array}$ \\
\hline $\begin{array}{l}\text { Study group } \\
\text { Conventional }\end{array}$ & 41 & 1 & 1 & 0 & $4.88 \%$ \\
group & 41 & 2 & 2 & 3 & $17.07 \%$ \\
$\mathrm{X}^{2}$ & - & & & & 13.082 \\
$\mathrm{p}$ & - & & & & $<0.05$ \\
\hline
\end{tabular}

\section{Discussion}

Anterolateral approach is a new type of minimally invasive approach in modern clinical practice. It requires a small incision diameter and attracts clinical attention due to it will not damage the surrounding structure around the joint [4]. The advantages of minimally invasive anterolateral approach include: (1) it does not need to cut off the muscle, which is helpful for patients to get out of bed as soon as possible after surgery and further accelerate the recovery process of hip joint function; (2) the incision approach is located at the tensor fascia lata and the gluteus medius muscle, and thus it will not damage the surrounding muscle group and is relatively minimally invasive; (3) the incision is small, and it is not easy to leave scars, which is easy for patients to accept; (4) short hospital stay can reduce the treatment burden of patients [5].

In this study, although the operation time of the study group was longer than that of the conventional group, the operation incision was smaller than that of the conventional group, and the operation bleeding volume and hospital time was lower than that of the conventional group ( $\mathrm{P}<$ 0.05). It shows that the anterolateral approach to total hip arthroplasty can better reduce the trauma to patients and accelerate the recovery speed of patients. However, the operation time is too long, 
which may be due to the fact that the surgeons are not familiar with this approach. With the increasing number of similar cases, it is believed that the surgery time will be shortened [6]. Harris hip function scores at the third, sixth and twelfth months of surgical treatment in the study group were higher than those in the conventional group $(\mathrm{P}<0.05)$. The results show that the anterolateral approach plays a more positive role in promoting the improvement of hip joint function. On the other hand, surgical complications in the study group were less than those in the conventional group (P $<0.05)$. The safety of anterolateral approach was fully confirmed. It should be mentioned that this operation is not suitable for all patients. Contraindications and indications should be strictly controlled before the operation. The anterolateral approach is not recommended for patients with high body mass index, over developed muscles, poor hip joint development, previous operation history and severe hip trauma [7].

Generally speaking, anterolateral approach to total hip arthroplasty has better clinical efficacy and is worthy of clinical application as the preferred method, with advantages of minimally invasive, fewer complications, faster recovery after surgery, etc.

\section{References}

[1] Huang Guofu, Su Fujin, He Zhong, et al. Early Curative Effect Analysis of Minimally Invasive Direct Anterior and Posterolateral Approach in Primary Total Hip Arthroplasty [J]. Sichuan Journal of Physiological Sciences, 2020, 42(03): 285- 289+305.

[2] Li Yongwang, He Rongli, Zhang Qian, et al. Comparison of Total Hip Arthroplasty between Direct Anterior Approach and Posterolateral Approach in the Aupine Position [J]. Chinese Tissue Engineering Research, 2020, 24(18): 2848-2854.

[3] Deng Chen, Ni Chaomin, Luo Zhengliang, et al. Comparative Study on the Difference between the Lateral Position of Direct Anterior Approach and Posterolateral Approach for Early Balance and Proprioception after Total Hip Arthroplasty [J]. Journal of Anhui Medical University, 2019, 54(11): 1795-1799.

[4] Jia Jian, Cheng Kai. Comparative Study on the Efficacy of Different Surgical Approaches for Total Hip Arthroplasty on Acetabular Dysplasia [J]. Chinese Medical Innovation, 2019, 16(23): 6-10.

[5] Zhu Ruixia, Zhao Gongyin, Wang Liangliang, et al. Comparison between Direct Anterior and Posterolateral Approach for the Treatment of Femoral Neck Fractures in Elderly Patients with Artificial Hip Arthroplasty [J]. Chinese Journal of Trauma, 2019(08): 730-735.

[6] Zhang Yuxin. Comparison of Clinical Efficacy between Direct Anterior Approach and Conventional Posterior Approach in Total Hip Arthroplasty [J]. Digest of World Latest Medical Information, 2018, 18(93): 39.

[7] Sun Baofei, Zhang Jingdong, Wang Danni, et al. Early Curative Effect Analysis of Direct Anterior Approach and Posterolateral Approach in Lateral Position in Primary Total Hip Arthroplasty [J]. Chinese Journal of Clinical Practical Medicine, 2017(03): 15-19. 